\title{
Comparison of conscious sedation and general anesthesia for motor mapping and resection of tumors located near motor cortex
}

\author{
Todd W. Vitaz, M.D., William Marx, M.D., Jonathan D. Victor, M.D., Ph.D., \\ AND PHILIP H. GuTIN, M.D. \\ Department of Neurological Surgery, University of Louisville School of Medicine, Louisville, \\ Kentucky; Neurosurgical Service and Department of Anesthesia, Memorial Sloan-Kettering Cancer \\ Center; and the Departments of Neurology and Neuroscience and Neurological Surgery, Weill \\ Medical College of Cornell University, New York, New York
}

\begin{abstract}
Object. The surgical treatment of tumors located near eloquent cortex carries a high risk of inducing worsening neurological deficits. Intraoperative electrocorticography techniques have been developed to help identify these areas at the time of surgery in an effort to minimize such risks. The optimal anesthetic technique for conducting these procedures, however, has never been determined.

Methods. The authors conducted a retrospective study to compare patients who underwent intraoperative motor mapping between September 2000 and May 2002. Demographic and neurophysiological monitoring data were collected from the hospital records. Patients were divided into two groups based on the anesthetic technique used for surgery: in Group 1 general anesthesia was used, and in Group 2 conscious sedation.

Group 1 comprised 24 patients (mean age 47 years) with 16 right- and eight left-sided lesions. Group 2 consisted of 21 patients (mean age 46 years) with 18 right- and three left-sided lesions. Pathological diagnoses were similar between the two groups. Motor stimulation was elicited in 12 patients $(50 \%)$ in Group 1 and in 21 patients $(100 \%)$ in Group $2(\mathrm{p}<0.001)$. In addition, the mean stimulation amplitude required was significantly higher $(13 \mathrm{~mA})$ in patients in whom conscious sedation was used as opposed to general anesthesia $(5 \mathrm{~mA}, \mathrm{p}<0.0001)$. Electrographic evidence of seizures was seen in $29 \%$ of Group 1 cmpared with $10 \%$ of Group 2 patients ( $\mathrm{p}>0.05)$.

Conclusions. The use of conscious sedation as an anesthetic technique for motor mapping not only improves the chances of achieving successful stimulation and identification of motor cortex in relationship to the lesion, but it also allows for repetitive monitoring of the patient's motor function during resection of the lesion.
\end{abstract}

\section{KEY WORDS • brain tumor • intraoperative mapping • awake craniotomy • anesthesia - motor cortex}

The primary goal for resection of brain tumors has been maximal tumor resection while minimizing the risks of postoperative neurological deficits. In cases in which lesions abut or invade motor and speech centers, however, the ability of the surgeon to achieve these goals becomes progressively more difficult. ${ }^{4,8,17}$ Numerous technological advances have been developed in an attempt to make safer the resection of lesions located near functionally important cortex. These include systems designed to localize functional cortex preoperatively such as fMR imaging, MEG, Wada testing, and intraoperative modalities designed to identify and differentiate tumor from surrounding brain (intraoperative ultrasonography, surgical navigation systems, and intraoperative MR imaging). $3,10,11$

The gold standard for identifying functional cortex,

Abbreviations used in this paper: $\mathrm{ECS}=$ electrocortical stimulation; $\mathrm{fMR}=$ functional magnetic resonance; $\mathrm{MEG}=$ magnetoencephalograpy; SSEP = somatosensory evoked potential. however, has remained direct ECS at the time of surgery. ${ }^{2,4,8,17}$ This technique has been described in conjunction with both general and "alert" anesthesia (conscious sedation), with the latter being predominantly reserved for speech mapping. ${ }^{2,4,9}$ The development of newer anesthetic agents, however, has made "awake" craniotomy simpler and more tolerable for patients. ${ }^{6,7,14,16}$ As a result of these advances, we have tended over the past 2 years to perform more conscious sedation-assisted craniotomies. To date, no comparison has been performed to evaluate the effects of these two types of anesthesia on the success of intraoperative motor mapping; therefore, we conducted a retrospective review to address this issue.

\section{CLINICAL MATERIAL AND METHODS}

A retrospective review of the surgical case log was performed to identify all intracranial operations in which the senior author (P.H.G.) performed intraoperative electro- 
corticography between September 2000 and May 2002. Cases that included any form of speech mapping were excluded because this technique mandated the use of alert anesthesia. All patients were treated by a single attending neurosurgeon, thereby limiting variability in surgical and mapping techniques. Hospital records, operative notes, and neurophysiological reports were then reviewed to collect demographic data and monitoring results. Patients were then divided into two groups: those in whom surgery and mapping were performed after induction of general anesthesia (Group 1) and those in whom conscious sedation was used (Group 2). Direct intergroup comparison was then performed, and statistical analysis was conducted using the Student t-test and chi-square test.

\section{Anesthetic Technique}

Goup 1. Endotracheal general anesthesia was composed of inhalation agents combined with Versed and fentanyl in the absence of paralytics.

Goup 2. Patients sedation was induced via a propofol infusion. A nasal trumpet lubricated with lidocaine jelly was then inserted, and oxygen was infused throughout the procedure. A foley catheter lubricated with lidocaine jelly and an arterial line were also placed. A local field block (a 1:1 mixture of $50 \mathrm{ml}$ lidocaine $1 \%$ with epinephrine $[1: 100,000]$ and $50 \mathrm{ml}$ bupivicaine $0.5 \%$ with epinephrine $[1: 200,000]$ mixed with $9 \mathrm{ml}$ sodium bicarbonate) was infiltrated into all of the regional nerves supplying the scalp. In addition, the pin sites anticipated for the placement of the Mayfield head holder (Ohio Medical, Cincinnati, OH) were infiltrated with the same solution. Once in place, the pin sites were reinfiltrated to ensure adequate coverage. Finally, the patient was prepared and draped, making sure that the anesthesiologist had an adequate avenue to the patient's face throughout the procedure, and the planned incision was infiltrated with $0.5 \%$ lidocaine with epinephrine $(1: 200,000)$. The patient remained sedated (via the propofol infusion) until approximately 10 minutes prior to the planned mapping at which time the infusion was discontinued. Versed, fentanyl, and labetalol were administered as needed throughout the procedure to control pain, anxiety, and hypertension. Patients remained awake throughout the mapping and during critical portions of the resection as determined by the surgeon. Once tumor resection was completed, the propofol infusion was restarted to sedate the patient during the closure procedure.

\section{Mapping Technique}

The mapping technique was the same in both groups. After induction of general anesthesia or conscious sedation, monitoring needles for upper- and sometimes lowerextremity SSEPs and reference leads were inserted.

Following adequate exposure, an eight-panel subdural electrode was placed on the cortical surface spanning the area of the presumed central sulcus with a slightly oblique alignment from posteromedial to anterolateral. Somatosensory evoked potentials were then recorded with the area of phase reversal, which is usually consistent with the central sulcus and was marked with a sterile paper ticket (Fig. 1). After localization of the central sulcus, an Ojemann stimulator (Radionics, Burlington, MA) $(60 \mathrm{~Hz}$, bi-

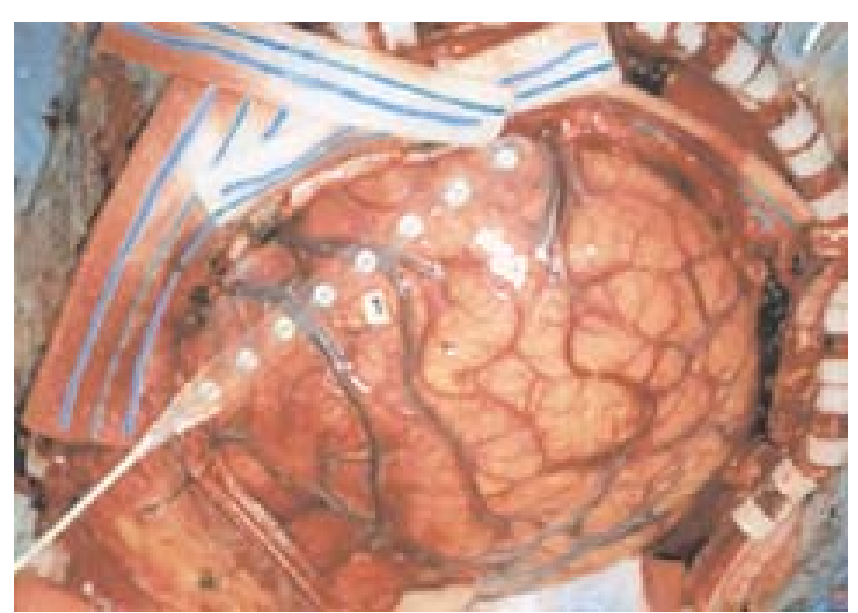

Fig. 1. Intraoperative photograph showing orientation of monitoring electrode for intraoperative SSEP. 1, Location of phase reversal.

phasic square wave, $1 \mathrm{msec}$ ) was used for direct ECS. During this procedure the subdural electrode was positioned at the edge of the operative field and used to monitor electroencephalography for the presence of afterdischarges or electrical seizure activity. Stimulation was typically initiated at an intensity of $4 \mathrm{~mA}$ (peak-to-peak) and increased as needed until a positive response or repetitive afterdischarges or clinical seizure activities were encountered. Once the motor cortex (both groups) or sensory cortex (awake group) was identified, the area over the region of the tumor was stimulated at a slightly higher intensity. All positive results were marked with numbered tickets (Fig. 2) and the mapping-related results as well as the surgical navigation system and intraoperative ultrasonography data were used to determine the best avenue for resection of the lesion. In circumstances in which the lesion abutted or was within the motor cortex, Group 2 patients were left alert and underwent intermittent motor function monitoring (hand grasp, dorsi-plantar flexion) until the resection was completed (Fig. 3).

\section{RESULTS}

The senior author performed resections in 45 patients with intracranial tumors located near motor cortex. Twenty-four patients underwent surgery after induction of general anesthesia (Group 1) and 21 after induction of conscious sedation (Group 2). Histological diagnosis, and tumor locations for the two groups are shown in Table 1. The use of SSEP confirmed localization an area of phase reversal in 83 and $86 \%$ of Groups 1 and 2 patients, respectively. Direct cortical stimulation elicited positive results in 12 of $24(50 \%)$ Group 1 patients and 21 of $21(100 \%)$ Group 2 patients $(\mathrm{p}<0.001)$. The mean intensities required to obtain these results were $13 \mathrm{~mA}$ (range 4-18 mA, median $16 \mathrm{~mA}$ ) in Group 1 and $5 \mathrm{~mA}$ (range 4-10 mA, median $4 \mathrm{~mA})$ in Group $2(\mathrm{p}<0.0001)$. Finally, there was a trend toward higher seizure incidence in Group 1 patients, although this did not reach statistical significance (29\% [Group 1] and 10\% [Group 2], p = 0.14). These results are shown in Table 2. 

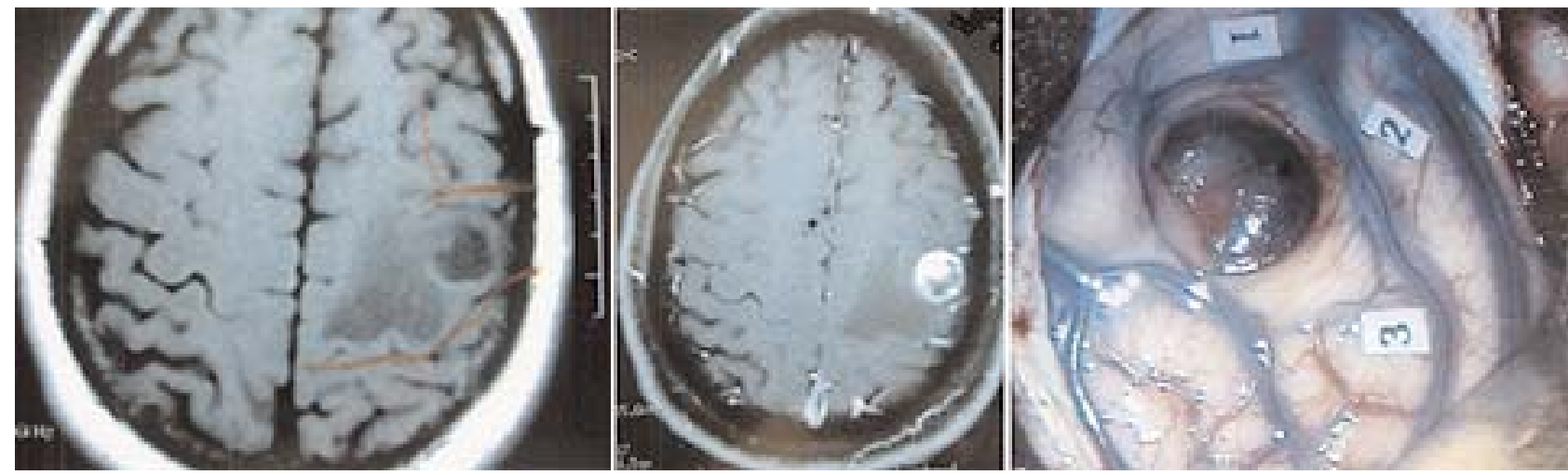

Fig. 2. Imaging studies obtained in a patient with metastatic adenocarcinoma. The patient refused stereotactic radiosurgery and instead opted for resection with awake mapping. Left and Center: Preoperative axial contrast-enhanced (left) and noncontrast (center) MR images. The red lines indicate the central sulcus based on radiographic landmarks. Right: Intraoperative photograph showing the location of tumor within sensorimotor cortex. Paper tickets identify areas of positive stimulation. 1 , motor for thumb; 2 , sensory for hand; 3 , motor for face.

\section{DISCUSSION}

Enormous effort has recently gone into developing noninvasive techniques for preoperative localization of eloquent portions of the brain. The various modalities range from topographic measurements and radiographic landmarks to fMR imaging and MEG. ${ }^{3,10,11}$ Their ability to localize motor cortex adequately increases from 50 to $67 \%$ when topographic landmarks are used, from 80 to $97 \%$ when fMR imaging is performed, and from 85 to $100 \%$ when MEG is conducted. ${ }^{3,8,10,11}$ Although these modalities in their current state may help to differentiate between cases requiring mapping-assisted tumor resection and those not, the gold standard remains intraoperative direct ECS.

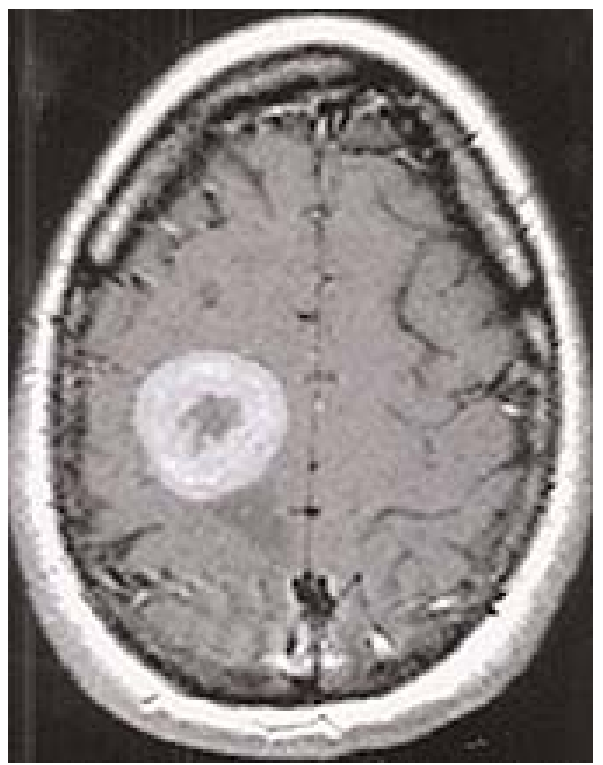

Fig. 3. Preoperative axial postcontrast MR image obtained in a patient with metastatic sarcoma who had previously undergone a right lower-extremity amputation; the lesion was located in the right motor cortex.
Historically, motor mapping has been performed after induction of general anesthesia; however, we have shown that these procedures can also be performed safely after administration of conscious sedation without any significant anesthesia-related perioperative complications. The biggest concern related to this technique includes loss of an adequate airway and subsequent failure to control hypercapnia-induced brain swelling; however, this is typically not a significant problem with an incidence of less than $1 \%$ in most studies. ${ }^{6,9,12,16}$ Likewise, we have not found this to be a significant problem, and brain swelling can typically be controlled using a combination of perioperative mannitol and dexamethasone (with furosemide being reserved for resistant cases).

The success of motor mapping depends highly on the depth of anesthesia and may be difficult when general anesthesia is administered (50\% in our study). We do not believe that the absence of stimulation in the operative field correlates with the absence of functional cortex in the

TABLE 1

Summary of data in 45 patients undergoing tumor resection

\begin{tabular}{lcc}
\hline \hline \multicolumn{1}{c}{ Characteristic } & $\begin{array}{c}\text { Group 1 } \\
\text { (general anesthesia) }\end{array}$ & $\begin{array}{c}\text { Group 2 } \\
\text { (conscious sedation) }\end{array}$ \\
\hline $\begin{array}{l}\text { no. of patients } \\
\text { mean age in yrs (range) }\end{array}$ & 24 & 21 \\
sex & $46(28.4-76.5)$ & $47.2(28.2-79.5)$ \\
$\quad$ female & 8 & 5 \\
male & 16 & 16 \\
pathological entity (\%) & & \\
glioblastoma multiforme & $6(25)$ & $1(5)$ \\
anaplastic astrocytoma & $8(33)$ & $3(14)$ \\
low-grade glioma & $5(21)$ & $10(48)$ \\
metastasis & $5(21)$ & $5(24)$ \\
radiation necrosis & 0 & $1(5)$ \\
abcess & 0 & $1(5)$ \\
location & & \\
left & $8(33)$ & $3(14)$ \\
right & $16(67)$ & $18(86)$ \\
frontal & $19(79)$ & $17(81)$ \\
parietal & $5(21)$ & $4(19)$ \\
\hline
\end{tabular}


TABLE 2

Results of cortical mapping

\begin{tabular}{lcc}
\hline \multicolumn{1}{c}{ Variable } & $\begin{array}{c}\text { Group 1 } \\
\text { (general anesthesia) }\end{array}$ & $\begin{array}{c}\text { Group 2 } \\
\text { (conscious sedation) }\end{array}$ \\
\hline $\begin{array}{l}\text { SSEP localization (\%) } \\
\text { positive stimulation } \\
\text { no. of cases (\%) }\end{array}$ & $20(83)$ & $18(86)$ \\
mean amplitude (range) & $12(50)$ & $21(100)$ \\
electrical seizure activity (\%) & $7(29)$ & $5 \mathrm{~mA}(4-10 \mathrm{~mA})$ \\
\hline
\end{tabular}

area being stimulated and have encountered such cases in which the patient awoke with a neurological deficit. Only when the motor cortex can be adequately identified can the surgeon be confident of its relationship to the tumor. ${ }^{4,8,17}$ The higher stimulation amplitudes often required to induce positive stimulation after induction of general anesthesia (13 mA compared with $5 \mathrm{~mA}$ in conscious sedation-treated patients, $\mathrm{p}<0.0001)$ might also increase the risk of inducing seizure activity (29\% in Group 1 and 10\% in Group 2, p > 0.05), which may further hinder the chances for successful stimulation if it becomes generalized. Although general anesthesia is known to have superb antiepileptic properties, its depth must be sufficiently decreased to allow for positive stimulation. This low-level anesthesia combined with the higher amplitudes required to induce positive stimulation results may increase the possibility of eliciting seizure activity. Finally, the propofol that is used in the conscious sedation-treated patients is known to have antiepileptic properties, which may be further protective in this group. . $^{1,5,13,15}$ The development of seizure activity is important for two reasons: it can lead to airway problems and, if this activity becomes diffuse or generalized, it may impair further attempts at stimulation or patient interaction because of the postictal refractory state of the brain.

Finally, the ability to obtain direct intraoperative physiological feedback from the patient significantly lessens the risks of producing new neurological deficits. When intraoperative deficits are encountered, the surgeon can either modify the approach or cease resection to minimize the chance of further injury., ${ }^{2,12}$ Danks, et al., ${ }^{6}$ reported that of 33 patients who underwent awake mapping and developed a deficit intraoperatively only two suffered permanent deficits.

Although we have shown that conscious sedation may be superior to general anesthesia as an adjuvant during resection of lesions located near motor cortex, the former is not the anesthetic of choice in all patients. Patients are not considered candidates for awake anesthesia if profound preoperative neurological deficits are present, which may preclude eliciting a positive response to stimulation, nor in the presence of significant comorbidities, such as severe cardiac or pulmonary disease, or psychological disorders that may interfere with their ability to undergo such a procedure. In addition, this modality is not typically performed in children younger than 15 years of age because of emotional immaturity. Danks, et al., ${ }^{7}$ prospectively evaluated their patients' perceptions and pain levels during such procedures and found that $81 \%$ suffered amnesia with regard to most or all of the procedure. In addition, only $5 \%$ complained of severe pain and $10 \%$ stated they felt anxious or uncomfortable during part of the procedure; 18 of the 21 patients said they would undergo the procedure again if necessary.

Although we did not conduct this study to demonstrate the different effects of anesthetic methods on successful intraoperative mapping, the retrospective design prevents intergroup comparison of neurological and radiographical outcomes. Memorial Sloan-Kettering Cancer Center is a quaternary referral center and, as such, many of the patients were not available for long-term follow-up assessment of neurological status; additionally preoperative radiographs were unavailable for review in most cases because they were typically returned to the home institution postoperatively.

A frequent concern among surgeons when evaluating new techniques is their impact on the overall length of a procedure. Although an awake craniotomy requires an additional 5 or 10 minutes, this time is usually recovered later during the procedure. Conscious sedation-assisted mapping often proceeds at a much faster pace because successful stimulation is often achieved using very low stimulation parameters and because sensory stimulation can be reported, which helps direct the surgeon to the location of motor cortex.

\section{CONCLUSIONS}

Overall we believe that conscious sedation-assisted resection of lesions near motor cortex requiring electrocortical mapping is superior to general anesthesia in selected patients. Successful stimulation was obtained in $100 \%$ of the conscious sedation-treated patients with significantly lower thresholds $(5 \mathrm{~mA})$ compared with $50 \%$ positive stimulation in the general anesthesia-treated patients (13 $\mathrm{mA}$ ). Finally, the ability to obtain direct physiological feedback from the patient during the most critical portion of the resection empowers the surgeon by providing more information that allows maximization of tumor resection and minimization of neurological deficits.

\section{References}

1. Begemann M, Rowan AJ, Tuhrim S: Treatment of refractory complex-partial status epilepticus with propofol: case report. Epilepsia 41:105-109, 2000

2. Berger M: Functional mapping-guided resection of low-grade gliomas. Clin Neurosurg 42:437-452, 1995

3. Berger MS, Cohen WA, Ojemann GA: Correlation of motor cortex brain mapping data with magnetic resonance imaging. J Neurosurg 72:383-387, 1990

4. Black PM, Ronner SF: Cortical mapping for defining the limits of tumor resection. Neurosurgery 20:914-918, 1987

5. Brown L, Levin G: Role of propofol in refractory status epilepticus. Ann Pharmacother 32:1053-1059, 1998

6. Danks RA, Aglio LS, Gugino LD, et al: Craniotomy under local anesthesia and monitored conscious sedation for the resection of tumors involving eloquent cortex. J Neurooncol 49: 131-139, 2000

7. Danks RA, Rogers M, Aglio LS, et al: Patient tolerance of craniotomy performed with the patient under local anesthesia and monitored conscious sedation. Neurosurgery 42:28-36, 1998

8. Duffau H, Capelle L, Sichez J, et al: Intra-operative direct electrical stimulations of the central nervous system: the Salpetriere experience with 60 patients. Acta Neurochir 141:1157-1167, 1999 
9. Haglund MM, Berger MS, Shamseldin M, et al: Cortical localization of temporal lobe language sites in patients with gliomas. Neurosurgery 34:567-576, 1994

10. Hirsch J, Ruge MI, Kim KH, et al: An intergrated functional magnetic resonance imaging procedure for preoperative mapping of cortical areas associated with tactile, motor, language, and visual functions. Neurosurgery 47:711-722, 2000

11. Lehericy S, Duffau H, Cornu P, et al: Correspondence between functional magnetic resonance imaging somatotopy and individual brain anatomy of the central region: comparison with intraoperative stimulation in patients with brain tumors. J Neurosurg 92:589-598, 2000

12. Meyer FB, Bates LM, Goerss SJ, et al: Awake craniotomy for aggressive resection of primary gliomas located in eloquent brain. Mayo Clin Proc 76:677-687, 2001

13. Prasad A, Worrall BB, Bertram EH, et al: Propofol and midazolam in the treatment of refractory status epilepticus. Epilepsia 42:380-386, 2001

14. Silbergeld DL, Mueller WM, Colley PS, et al: Use of propofol
(Diprivan) for awake craniotomies: technical note. Surg Neurol 38:271-272, 1992

15. Stecker MM, Kramer TH, Raps EC, et al: Treatment of refractory status epilepticus with propofol: clinical and pharmacokinetic findings. Epilepsia 90:18-26, 1998

16. Taylor MD, Bernstein M: Awake craniotomy with brain mapping as the routine surgical approach to treating patients with supratentorial intraaxial tumors: a prospective trial of 200 cases. J Neurosurg 90:35-41, 1999

17. Yingling CD, Ojemann S, Dodson B, et al: Identification of motor pathways during tumor surgery facilitated by multichannel electromyogrhaphic recording. J Neurosurg 91:922-927, 1999

Manuscript received May 28, 2003.

Accepted in final form June 13, 2003.

Address reprint requests to: Todd W. Vitaz, M.D., 210 E. Gray Street, Suite 1102, Louisville, Kentucky 40202. email: Tvitaz@ NIKY.com. 